\title{
Quantitative heel ultrasound variables in powerlifters and controls
}

\author{
S Jawed, B Horton, T Masud
}

\begin{abstract}
Objective-To compare by cross sectional study the quantitative heel ultrasound (QUS) variables broadband ultrasound attenuation (BUA) and velocity of sound (VOS) in male powerlifters and controls. Methods-Twenty four powerlifters and 21 sedentary male controls were recruited to the study. All the powerlifters were members of the British Drug Free Powerlifting Association and actively competing at the time of the study. A questionnaire was completed by all those entered into the study. This included a history of smoking and an estimation of daily intake of alcohol and calcium. For the powerlifters, the number of years spent training and time spent training each week was also recorded. The QUS variables of all powerlifters and controls were measured using a Cubaclinical II (McCue) ultrasound scanner.
\end{abstract}

Results-The powerlifters had been training for a mean (SEM) of 10.6 (1.6) years and they trained for $6.5(0.4)$ hours a week. The powerlifters were non-significantly older and had a significantly higher body mass index (BMI) than the controls. Calcium intake and consumption of alcohol and tobacco were similar in the two groups. The mean BUA in the powerlifters was a significant $9.5 \%$ (95\% confidence interval 0.7 to $18.3 \%$ ) higher than the controls (105 $v$ $96 \mathrm{~dB} / \mathrm{MHZ})$ and $15.6 \%$ (95\% confidence interval 6.8 to $24.4 \%$ ) higher after adjustment using analysis of covariance for age, BMI, and alcohol and tobacco consumption (108 $v 93 \mathrm{~dB} / \mathrm{MHZ}$ ). The mean VOS was similar in the two groups, but after adjustment it was significantly higher in the powerlifters $(1671 v 1651 \mathrm{~m} / \mathrm{s}, \mathrm{p}<0.01)$. Conclusions-The study shows the ability of heel ultrasound to discriminate between QUS variables in powerlifters and controls. The results indicate that the QUS variables BUA and VOS are significantly higher for powerlifters than for controls.

(Br F Sports Med 2001;35:274-275)

Keywords: osteoporosis; bone; heel; ultrasound; powerlifter

Osteoporosis is now a major public health problem consuming vast resources. ${ }^{1}$ Although rapid progress is being made in developing new treatments for established disease, the best long term strategy is likely to remain prevention. One of the modifiable risk factors is exercise, which can improve bone mineral density
(BMD), which in turn may reduce future risk of osteoporotic fracture. Of all types of exercise, the most promising results have been with resistance exercise, which appears to increase BMD considerably. ${ }^{2}$ The effect on BMD of different types of resistance exercise regimens varies in different population groups. ${ }^{3-5}$ Powerlifting is an example of extremely high intensity resistance exercise aimed at developing maximum strength in three specific weight training exercises: deep knee bend (squat), bench press, and deadlift. Existing small studies using dual energy $x$ ray absorptiometry have shown that young male powerlifters have increased lumbar spine and whole body $\mathrm{BMD},{ }^{6}$ and that junior Olympic lifters have a higher lumbar spine and femoral neck BMD. ${ }^{7}$ In addition to dual energy $x$ ray absorptiometry measurements of BMD, low quantitative heel ultrasound (QUS) variables are themselves a predictor of increased fracture risk. $^{8-10}$ In this study we investigate the association of powerlifting with the QUS variables broadband ultrasound attenuation (BUA) and velocity of sound (VOS). The aim was to compare these variables in male powerlifters and controls.

Methods and subjects

Ethical approval was obtained for this study and written consent obtained from all volunteers. Twenty four powerlifters and 21 sedentary controls who took no regular exercise were recruited to the study. All the powerlifters were members of the British Drug Free Powerlifting Association (BDFPA) and actively competing at the time of the study. The BDFPA has a rigorous programme of in competition and zero notice out of competition drug testing, which tests for all substances banned by the International Olympic Committee. Those tested positive receive an automatic lifetime ban from future competitions. All the powerlifters had competed for at least two years and were all of national standard.

Height and weight were measured in all subjects. A questionnaire was also completed by all those entered into the study. This included a history of smoking and an estimation of daily intake of alcohol and calcium. Calcium intake was scored from 1 to 3 : 1 , less than $1 \mathrm{~g}$ dietary calcium a day; 2, 1-2 $\mathrm{g}$ a day; 3 , more than $2 \mathrm{~g}$ a day. For the powerlifters, additional information on number of years training and time spent training a week was also recorded. The QUS variables of all powerlifters and controls were measured using a Cubaclinical II (McCue) ultrasound scanner. Both BUA and VOS were recorded for all subjects. 
Table 1 Baseline characteristics of powerlifters and controls

\begin{tabular}{llll}
\hline & Powerlifters & Controls & p Value \\
\hline Number & 24 & 21 & - \\
Age (years) & $41(12)$ & $36(11)$ & 0.12 \\
BMI (kg/m $\left.{ }^{2}\right)$ & $30.2(4.0)$ & $27.0(2.4)$ & 0.002 \\
Alcohol intake & $8.0(8.8)$ & $11.5(3.1)$ & 0.29 \\
Cpd & $1.7(5.3)$ & $5.6(9.7)$ & 0.05 \\
Calcium (1-3) & $2.2(1.0)$ & $2.4(0.6)$ & 0.4
\end{tabular}

Values are mean (SEM). Alcohol intake is given in units per week. Calcium intake per day: $1=1 \mathrm{~g} ; 2=1-2 \mathrm{~g} ; 3=>2 \mathrm{~g}$.

$\mathrm{BMI}$, Body mass index; Cpd, cigarettes per day.

\section{STATISTICAL ANALYSIS}

The statistical package SPSS for windows (version 8.0) was used for analysis. Results are shown as mean (SEM). Analysis of covariance was used to adjust the results for age, body mass index (BMI), cigarette and alcohol consumption, and dietary calcium intake.

\section{Results}

Table 1 shows the baseline data for the two groups including the results of the questionnaire. The mean (SEM) length of time for which the powerlifters had been training was 10.6 (1.6) years, and they trained for $6.5(0.4)$ hours each week. The powerlifters were non-significantly older, and had a significantly higher BMI than the controls $(30.4(0.7)$ v 27.0 (2.4), p<0.01). Calcium intake was similar in the two groups. There was a nonsignificant trend towards lower consumption of alcohol and tobacco in the powerlifters.

Table 2 shows the actual QUS variables and adjusted values. The mean BUA in the powerlifters was a significant $9.5 \%$ (95\% confidence interval 0.7 to $18.3 \%$ ) higher than the controls $(105 v 96 \mathrm{~dB} / \mathrm{MHZ})$ and $15.6 \% \quad(95 \%$ confidence interval 6.8 to $24.4 \%$ ) higher after adjustment using analysis of covariance for age, BMI, and alcohol and tobacco consumption (108 $v 93 \mathrm{~dB} / \mathrm{MHZ}$ ). The mean VOS was similar in the two groups, but, after adjustment for the above covariates, it was significantly higher in the powerlifters (1671 $v 1651 \mathrm{~m} / \mathrm{s}$, $\mathrm{p}<0.01)$

\section{Discussion}

This study shows that the QUS variables BUA and VOS are significantly higher for powerlifters than for controls. Although the powerlifters trained for an average of 6.5 hours a week, the training typically consists of low repetitions with use of weights that are close to the lifters' single repetition maximum. The actual time spent performing resistance exercise during the training sessions is only a small proportion of the total training time, as long rest periods are required between exercises. The substantial
Table 2 Quantitative heel ultrasound variables in powerlifters and controls

\begin{tabular}{llll}
\hline & Powerlifters & Controls & p Value \\
\hline BUA (dB/MHz) & $105(16)$ & $96(14)$ & 0.048 \\
BUA (\%) & $113(14)$ & $99(14)$ & 0.006 \\
Adjusted BUA & $108(16)$ & $93(14)$ & 0.026 \\
VOS (m/s) & $1663(32)$ & $1660(30)$ & 0.74 \\
VOS (\%) & $106(2)$ & $104(8)$ & 0.15 \\
Adjusted VOS & $1671(16)$ & $1651(30)$ & 0.008 \\
\hline
\end{tabular}

Values are mean (SEM).

BUA, Broadband ultrasound attenuation; VOS, velocity of sound.

improvement in QUS variables seems to occur with a limited amount of resistance exercise provided that the exercise is of high intensity.

In a previous study of 81 women, the CUBA clinical QUS system showed a significant correlation between level of activity and QUS variables. ${ }^{11}$ Ultrasound has also been used to show increased QUS variables in young male gymnasts. ${ }^{12}$ The results of this study add to information gained from previous studies. We have shown the ability of heel ultrasound to discriminate between QUS variables in powerlifters and controls, and the results obtained support the role of resistance exercise in improving bone QUS variables.

We would like to thank the BDFPA for their cooperation in allowing us to recruit lifters for this study during their competitions.

1 Cummings SR, Kelsey JL, Nevitt MC, et al. Epidemiology of osteoporosis and osteoporotic fractures. Epidemiol Rev 1985;7:178-208

2 Chilibeck PD, Sale DG, Webber CE. Exercise and bone mineral density [review]. Sports Med 1995;19:103-22.

3 Nichols JF, Nelson KP, Peterson KK, et al. Bone mineral density responses to high-intensity strength training in active older women. Fournal of Ageing and Physical Activity 1995;3:26-38

4 Gleeson PB, Protas EJ, LeBlanc AD, et al. Effects of weight lifting on bone mineral density in premenopausal women. $\mathcal{F}$ Bone Miner Res 1990;5:153-8.

5 Colletti LA, Edwards J, Gordon L, et al. The effects of muscle building exercise on bone mineral density of the radius, spine and hip in young men. Calcif Tissue Int 1989;45:1214.

6 Tsuzuku S, Ikegami Y, Yabe K. Effects of high-intensity resistance training on bone mineral density in young powerlifters. Calcif Tissue Int 1998;63:283-6.

7 Conroy BP, Kraemer WJ, Maresh CM, et al. Bone mineral density in elite junior Olympic weightlifters. Med Sci Sports density in elite junior Olyn

8 Huang C, Ross PD, Yates AJ, et al. Prediction of fracture risk by radiographic absorptiometry and quantitative by radiographic absorptiometry and quantitative ultrasounc

9 Hans D, Dargent-Molina P, Schott AM, et al. Ultrasonographic heel measurements to predict hip fracture in elderly women: the EPIDOS prospective study. Lancet 1996;348:511-14

10 Bauer DC, Gluer CC, Cauley JA, et al. Bone ultrasound predicts fractures strongly and independently of densitometry in older women: a prospective study. Arch Intern Med 1997;157:629-34

11 Messenger N, Scott S, McNaught-Davis P. Can the effects of exercise on bone quality be detected using the CUBA clinical ultrasound system? Brf Sports Med 1998:32:162-6.

12 Daly RM, Rich PA, Klein R. Influence of high impact loading on ultrasound bone measurements in children: sectional report. Calcif Tissue Int 1997;60:401-4.

\section{Take home message}

Quantitative heel ultrasound measurements are higher in powerlifters than normal sedentary controls. This suggests that high intensity resistance exercise has a positive effect on bone, but a prospective study is needed to confirm this. 